\title{
Impact of resistant starch type-3 on glucose metabolism and appetite in healthy males
}

\author{
M. H. Alhussain ${ }^{1}$, A. Almousa ${ }^{1}$ and A. Alhowikan ${ }^{2}$ \\ ${ }^{1}$ Department of Food Science and Nutrition, College of Food and Agriculture Sciences, King Saud University, \\ Saudi Arabia and \\ ${ }^{2}$ Department of Physiology, College of Medicine, King Saud University, Saudi Arabia
}

Introduction: Resistant starch (RS) has been considered to have many of the health benefits of dietary fibre ${ }^{(1-2)}$. Although resistant starch type-3 (retrograded starch, RS3) has a particular interest, due to its thermal stability where it has high gelatinization temperatures, a very limited number of studies have investigated its beneficial impact on health ${ }^{(3-4)}$. The current study aimed to examine the effects of RS3 on postprandial glycemic and insulinemic responses as well as appetite in young healthy males.

Methods: Eight healthy males aged 18-35 years, BMI $23.9 \pm 0.8 \mathrm{~kg} / \mathrm{m} 2$, participated in this randomized, single-blind crossover control study. Participants attended the laboratory after overnight fasting ( $\geq 8 \mathrm{~h})$ on two separate occasions, at least 1 week apart. On each laboratory visit, participants were given either a control meal or an RS3 meal. Both of the two meals were vegetarian pasta and were matched for energy intake and composition, ingredients and amount but were prepared in a different way. The control meal was hot freshly cooked pasta and the RS3 meal was re-heated pasta that had been chilled overnight. Blood samples were taken at fasting and 15-min intervals for $3 \mathrm{~h}$ after the meal consumption. Subjective appetite ratings were also assessed using visual analogue scale (VAS) while fasting and $3 \mathrm{~h}$ following the meal consumption. Differences in postprandial blood variables and subjective appetite ratings were determined.

A significant increase in iAUC for satiety following the RS3 meal consumption compared with the control meal was observed (mean \pm SD: RS3 meal: $10181.25 \pm 3140.65 \mathrm{~mm}$; control meal: $7132.37 \pm 3187.31 \mathrm{~mm}$; $\mathrm{p}=0.03$, paired T-test). iAUC for desire to eat was significantly decreased following the RS3 meal consumption compared with the control meal $(-8190.75 \pm 4333.34 \mathrm{~mm}$ vs $-4594.50 \pm 1481.11 \mathrm{~mm} ; \mathrm{p}=0.03$, paired T-test). On the other hand, no significant differences were found in postprandial blood glucose, serum insulin and plasma ghrelin levels between the two meals (All $\mathrm{p}>0.05$, paired T-test). There were also no differences in fasting blood glucose, serum insulin, plasma ghrelin and subjective appetite ratings between the control and RS3 meals (All $\mathrm{p}>0.05$, paired T-test).

This study shows that consuming the RS3 meal was associated with greater satiety and lower desire to eat in young healthy males which may lead to weight management in the long term. Further intervention studies are warranted to examine the beneficial impact of long-term consumption of RS3 on health.

\section{References}

1. Champ, M (2004) J AOAC Int 87, 749-755.

2. Lin CH, Chang DM, Wu DJ et al. (2015) Medicine 94 (33).

3. Lobley GE, Holtrop G, Bremner DM et al. (2013) Nutrients 5, 2144-2172.

4. Sajilata MG, Singhal RS \& Kulkarni PR (2006) Compr Rev Food Sci Food Saf 5, 1-17. 